\title{
Modelling the CAMP pathway using BioNessie, and the use of BVP techniques for solving ODEs Xu Gu*1, David Gilbert ${ }^{1}$, Des Higham ${ }^{2}$ and Miles Houslay ${ }^{3}$
}

\author{
Address: ${ }^{1}$ Bioinformatics Research Centre, University of Glasgow, Scotland, UK., ${ }^{2}$ Department of Mathematics, University of Stratheclyde, \\ Scotland, UK. and ${ }^{3}$ Institute of Biomedical and Life Sciences, University of Glasgow, Scotland, UK. \\ Email: Xu Gu* -gux@brc.dcs.gla.ac.uk \\ * Corresponding author
}

from BioSysBio 2007: Systems Biology, Bioinformatics and Synthetic Biology Manchester, UK. II-13 January 2007

Published: 8 May 2007

BMC Systems Biology 2007, I(Suppl I):P6 doi:10.1 I86/I752-0509-I-SI-P6

This abstract is available from: http://www.biomedcentral.com/I752-0509/I?issue=SI

(c) 2007 Gu et al; licensee BioMed Central Ltd.

Biochemists often conduct experiments in-vivo in order to explore observable behaviours and understand the dynamics of many intercellular and intracellular processes. However an intuitive understanding of their dynamics is hard to obtain because most pathways of interest involve components connected via interlocking loops. Formal methods for modelling and analysis of biochemical pathways are therefore indispensable. To this end, ODEs (ordinary differential equations) have been widely adopted as a method to model biochemical pathways because they have an unambiguous mathematical format and are amenable to rigorous quantitative analysis. BioNessie http://www.bionessie.com is a workbench for the composition, simulation and analysis of biochemical networks which is being developed in by the Systems Biology team at the Bioinformatics Research Centre as a part of a large DTI funded project 'BPS: A Software Tool for the Simulation and Analysis of Biochemical Networks' http://www.brc.dcs.gla.ac.uk/projects/dti beacon.

BioNessie is written in Java using NetBeans Platform libraries that makes it platform independent. The software employs specialised differential equations solvers for stiff and non-stiff systems to produce model simulation traces. BioNessie provides a user-friendly interfact that comes up with an intuitive tree-based graphical layout, an edition function to SBML-compatible models and feature of data output.
cAMP is an acknowledged second messenger which is involved in mediating the action of a wide range of processes in specific cells, for example the control of various metabolism events, gene expression, cell growth and division and cell apotosis, muscle contraction, secretion and memory, for example [1]. Part of the aims of Xu's PhD project are to use ODE modelling techniques to investigate the effects of different phosphodiesterase on cAMP degradation, functions of $\beta$-arrestin on uncoupling G-protein-coupled receptor and connections between cAMP pathway and the ERK pathway, for which no computational model currently exists. The interaction between these two signalling pathways is of pivotal importance and is crucial to the understanding of cell function and thus in developing new diagnostic methodologies and new therapeutics. I have used the software tool Bionessie to creat an SBML model of the CAMP signalling pathway based on data reported by Bhalla [2]. The behavioural dynamics of cAMP was fully examined using computational simulations in Bionessie (see figure 1), and an error was found in CAMP-PKA module of the pathway.

The computational modelling of signalling pathways using ODEs relies on exact values for rate constants and initial concentrations; however, the lack of sufficient data seriously limits the use of this approach. Thus a further aim of Xu's project is to address this challenge by investigating methods to permit users to explore the range of possible solutions for a model by taking into account ine- 


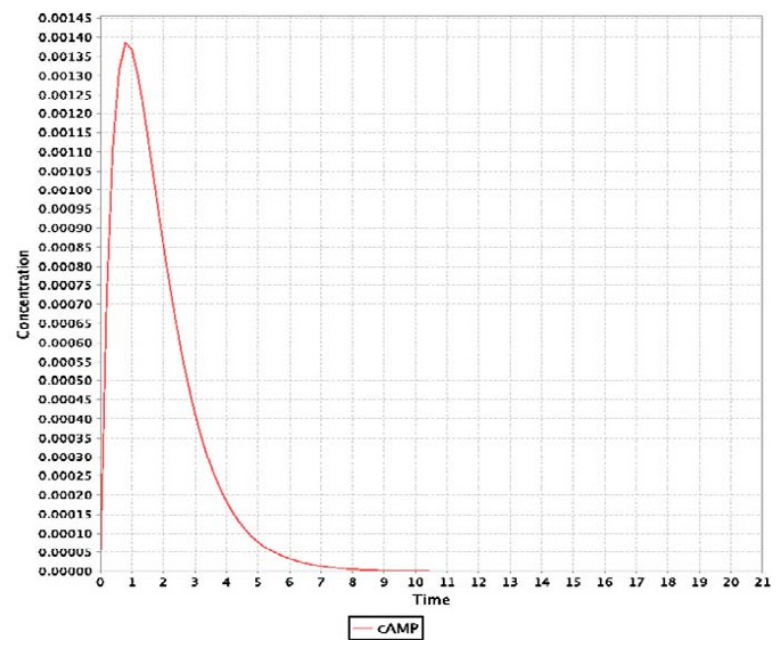

Figure I

cAMP pathway is a good example of signal amplification: one hormone molecule can lead to formation of many cAMP molecules. Low levels of cAMP allow large fractional increase and decrease in the concentration of cAMP with small amounts of CAMP made or broken down, so that the activation and deactivation can be rapid. As depicted here, cAMP increases to the peak soon as it is activated and then degraded dramatically at around 10 seconds. The figure was produced with the BioNessie.

quality constraints in order to permit an ODE solver to operate over partial data. Towards the end, I have implemented a two-point BVP (Boundary Value Problem) solver in Matlab, for the solution of problems where only partial data is vailable. In contrast to other BVP solvers that require exact boundary value conditions to be provided, this solver can solve inequality constraints and plot solutions with certain error distributions (see figure 2). While not in a polished form, the use of the solver illustrates some of the theoretical and practical aspects os this problem, and demonstrates the usability and flexibility of the preliminary version. To summarise, the resulting project will produce a powerful analytical tool for rapid and extensive analysis and testing of models of biochemical networks in order to provide novel hypotheses that can then be evaluated experimentally with data generated by Professor Houslay's wet laboratory.

\section{References}

I. Miles Houslay D, Walter Kolch: Cell-type specific integration of cross-talk between extracellular signal-regulated kinase and camp signalling. Molecular Pharmacology 2000, 58:659-668.

2. Upinder Bhalla S: Use of kinetikit and genesis for modeling signaling pathways. Methods in Enzymology 2002, 345:3-23.

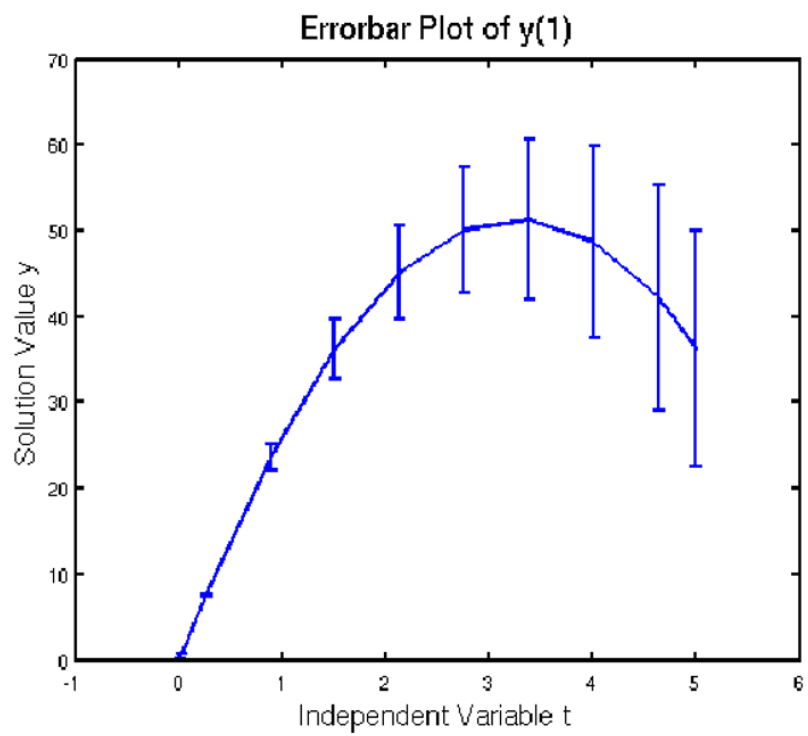

Figure 2

The solver is based on a shooting approach and programmed in Matlab. It employs the Secant method for a root finding and enables to solve separted and Dirichlet boundary conditions. The solver can also handle inequality constraints (e.g. a range of values) and plot solutions with certain error distributions.
Publish with Biomed Central and every scientist can read your work free of charge

"BioMed Central will be the most significant development for disseminating the results of biomedical research in our lifetime. " Sir Paul Nurse, Cancer Research UK

Your research papers will be:

- available free of charge to the entire biomedical community

- peer reviewed and published immediately upon acceptance

- cited in PubMed and archived on PubMed Central

- yours - you keep the copyright

Submit your manuscript here:

http://www.biomedcentral.com/info/publishing_adv.asp 